\section{Die am häufigsten gestellten Fragen zu TarMed}

Wie errechnet sich das Assistenz-Honorar?

K. G. in $S$.

In \%-Ansätzen des Honorars des Operateurs, abgestuft nach quantitativer Dignität der operativen/interventionellen Leistung, für der/die AssistentIn assistiert.

Ich mache als Arzt für Allgemeinmedizin FMH bei einem Arzt in Deutschland berufsbegleitend eine in der Schweiz nicht anerkannte Ausbildung in psychosomatischer Medizin. Kann ich diese psychosomatischen Beratungen in irgendeiner Form über den TarMed abrechnen oder kann ich dies nur privat verrechnen?

R. H. in B.

Nein. Die deutsche Ärztekammer hat eine gegenseitige Anerkennung von schweizerischen und deutschen Weiterbildungsausweisen abgelehnt. Eine
Änderung könnte - allenfalls! - eine Annahme der bilateralen Verträge bringen; im Detail wäre dann aber noch zu überprüfen, ob für Ihren Ausweis eine schweizerische Anerkennung möglich wäre.

Interpretiere ich den Tarif richtig, wenn ich zum Schluss komme, dass neu die maximal verrechenbare Konsultationszeit für "gewöhnliche" Konsultationen 17,5 Min. beträgt? Aufwessen Verlangen wurde diese doch einschneidende Limitierung eingeführt?

T. G. in $B$.

Gilt nur für den nicht auf elektronischem Datenträger abrechnenden Arzt. Für eine adäquate Beurteilung sind aber alle Leistungspakete, besonderen Tarifpositionen (Arbeit in Abwesenheit des Patienten, Psychotherapie, etc.) zu berücksichtigen. Wir empfehlen Ihnen eine entsprechende Durchsicht des Kapitels 0 unter Berücksichtigung Ihres Facharzttitels, der aus Ihrem Schreiben nicht zu entnehmen ist.

Wie hoch ist eigentlich der Taxpunktwert?

A. W. in $T$.

Wissen wir noch nicht. Abhängig von den kantonalen Verhandlungen für den KVG-Bereich. Im UV/MV/ IV-Bereich wird die Festlegung bis Mitte Jahr erfolgen (schweizerischer Einheitstarif mit einheitlichem Taxpunktwert ganze Schweiz).

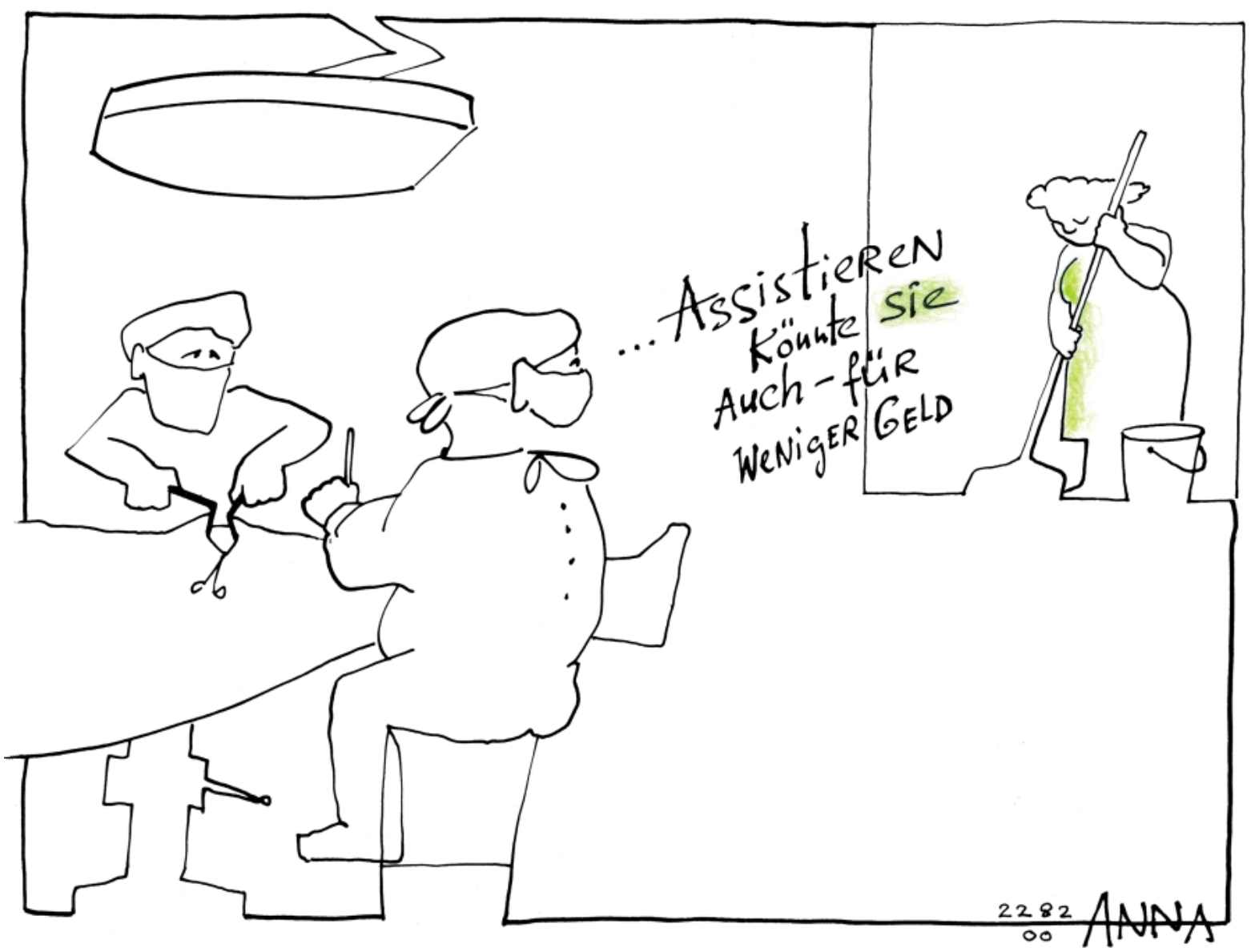


Wir betreiben eine orthopädische Gemeinschaftspraxis mit integrierter, ärztlich geleiteter Physiotherapie. Bislang vermisse ich im TarMed den physiotherapeutischen Teil. Wird dieser bis zur Einführung am 1.7. integriert sein oder ist dies gar nicht geplant?

Werden wir dann zum Tarif der Physiotherapeuten mit TPW 1 sFr. abrechnen oder wie soll das laufen? Es wäre schön, diese Antwort bald zu erhalten, die Kündigungsfrist unserer Therapeuten beträgt 3 Monate!

W. N. in $K$.

Physiotherapeutische Leistungen werden nach dem Physiotherapietarif zum in Ihrem Kanton gültigen Taxpunktwert abgerechnet; sie sind deshalb in der TarMed-Struktur nicht enthalten. Offen bleibt die Abgeltung der komplex rehabilitativen Physiotherapie; Stichwort für Eingeweihte: TarReha.

Ich untersuche den Patienten. Dann schicke ich ihn ins Röntgenzimmer. Wenn das Bild fertig ist, hole ich den Patienten wieder im Wartezimmer: Dauer 10 Min. +30 Min. + 10 Min.

Ist dies eine Konsultation à 50 Minuten: oder sind dies 2 Konsultationen à 10 Minuten (also $2 \times$ erste Kons.); oder ist dies eine Konsultation à 20 Minuten (das Röntgen wird nicht hinzugezählt).

A. W. in B.

Eine sogenannte Sitzung (vgl. allg. Interpretationen in TarMed Alpha 2.2) dauert vom Moment, in dem der Patient die Praxis betritt bis er sie wieder verlässt. Verrechnet wird die Zeit, während der der Arzt sich mit dem Patienten medizinisch beschäftigt, dies durch Konsultationstranchen à 5 Minuten. Die Arztzeit für Extraleistungen, z.B. Röntgen, wird über die entsprechende Extraleistungsposition abgerechnet; während dieser Zeit darf keine Konsultationszeit verrechnet werden. Dies gilt selbstverständlich auch für Zeit ohne ärztliche Betreuung oder Untersuchungen, also während der Patient zum Beispiel im Wartezimmer auf weiteren ärztlichen Zuspruch wartet. Könnte diese Zeit auch verrechnet werden, müssten wir uns über die finanzielle Zukunft der ÄrztInnen kaum Gedanken machen, sehr wohl aber über diejenige der Versicherer. Ist nicht ernst gemeint, wie auch wohl Ihre Frage nicht.
In den FAQ zu TarMed wird die Frage der Berichte von R. S. in A. aufgegriffen und beantwortet. (Randbemerkung: das swiss english hat schon seine Tücken, was soll das F in FAQ bedeuten, handelt es sich doch hier um eine Rubrik für AQ?) 00.1580 et analoga: "vom Versicherer nur entschädigt, wenn auf dessen Verlangen zugestellt."

Ich bin erschrocken über die mehrdeutige Formulierung, die nach Annahme des Vertrages ja in ihrer Mehrdeutigkeit Gültigkeit hat:

Interpretationsmöglichkeiten:

- "Vom Versicherer nur entschädigt, wenn auf dessen Verlangen ihm eine Kopie zugestellt wird"

- "Vom Versicherer nur entschädigt, wenn auf dessen Verlangen verfasst und zugestellt wird"

- "Vom Versicherer nur entschädigt, wenn auf dessen Verlangen verfasst und mit einer Kopie an ihn zugestellt wird"

Nicht interpretiert werden kann, dass Berichte, die keinem Verlangen des Versicherers entsprechen, entschädigt werden. Im KK-Bereich sind viele sehr sensible Persönlichkeitsdaten für eine korrekte Behandlung eines ganz gewöhnlichen Kranken nötig, Familienanamnese, somatische, psychische und soziale Gegebenheiten u.a.m. Dem Versicherer können sie zu Risikoabschätzung etc. dienen (auch Begriffe wie Vater, Mutter, Bruder 2 Jahre jünger etc. sind exakt zuzuordnen). Die Fragen um Geheimnisverwaltung sind ja mindestens in praxi via "vertrauensärztlicher Dienst" überhaupt nicht gelöst und verbergen einige Pulverfässer. Der Kranke gibt sie oft nur preis, weil er weiss, dass sie zum Gesundwerden wichtig sein können und weil er noch immer glaubt, sie seien wohl verwahrt ...

Sicher scheint mir, dass mit der gewählten Formulierung dem Arztgeheimnis eine weitere Baggerschaufel Erde auf den Grabeshügel geäufnet wurde. $\mathrm{Ob}$ dies die Betroffenen, um die es eigentlich gehen sollte, auch schätzen werden? Am ehesten wird Ihr Rechtsdienst die Frage beantworten können, auf welche Weise die Formulierung aus dem genehmigten Vertrag geworfen werden kann, ich könnte mir denken, dass es via Datenschutzgesetz ginge.

Gerne erbitte ich mir eine gelegentliche Stellungnahme, vielleicht bräuchte es ja einen Melder oder Kläger.

B. K. in $E$.

Hier handelt es sich um einen Fehler bzw. eine Unvollständigkeit. Selbstverständlich werden medizinisch indizierte, einen Patienten betreffende Schreiben nach den Positionen nicht formalisierte Berichte verrechnet. Dies wird auch ausdrücklich so festgehalten werden. 
Im Zusammenhang mit Ihrem Artikel «Umsetzung Dignität TarMed" in der Schweizerischen Ärztezeitung 2000, Heft Nr. 3, habe ich eine Frage. Ich bin Spezialarzt FMH für Psychiatrie und Psychotherapie. Gelegentlich assistiere ich zudem seit einigen Jahren einem operativ tätigen Kollegen. Muss ich nun, damit er meine Operationsassistenztätigkeit weiterhin über die Versicherungen oder die KK abrechnen kann, neu irgendwo, irgendetwas beantragen?

P. E. in Z.

Der Operateur ist in der Wahl seiner Assistenz völlig frei; er hat ausschliesslich für die notwendige Qualität persönlich zu garantieren. Besondere Anforderungen an die Qualifikationen des Assistenten bestehen nicht; so ist es unter anderem auch möglich, Nichtärztinnen bzw. Nichtärzte als Assistentinnen bzw. Assistenten zuzuziehen. Daraus ergibt sich auch, dass Sie Ihrerseits keine besonderen Massnahmen zu treffen bzw. Anträge zu stellen haben.

Ich habe per 1.1.2000 eine Praxis eröffnet (FMH Innere Medizin), als Ärztin führe ich auch gynäkologische Untersuchungen, transvaginale Ultraschalle und Schwangerschaftskontrollen durch. Werde ich dies auch nach Einführung des TarMed noch verrechnen können? Was heisst "regelmässig" in diesem Fall, da die Praxis erst am 1.1.2000 eröffnet wurde, reicht dies?

A. A. in $S$.

Hängt vom definitiven Inkrafttreten der Tarife ab; wahrscheinlich ja.
Ich bin seit mehreren Jahren an einem HMO-Zentrum tätig und führe entsprechend meiner Fort- und Weiterbildung dort regelmässig Zusatzuntersuchungen wie Ergometrie, Ultraschall des Abdomens aber auch Manuelle Therapie und Akupunktur durch. Diese Untersuchungen geschehen als angestellter Arzt aber nicht auf eigene Abrechnung. Was habe ich bezüglich Besitzstandswahrung nach Einführung des TarMed zu erwarten? Müssen wir unsere Abrechnungen ändern, damit der einzelne Leistungserbringer sichtbar wird? Was würde bei einer späteren Aufnahme einer selbständigen Tätigkeit passieren bezüglich meiner «wohlerworbenen Rechte»?

F. B. in $S$.

Die von Ihnen indirekt angesprochene Besitzstandswahrung dient der Sicherung eines selbständigerwerbenden Geschäftsinhabers, dessen Existenz nicht durch unvermittelte, gegen Treu und Glauben verstossende Änderungen politischer bzw. gesetzlicher oder verwandter Rahmenbedingungen vernichtet werden darf. Wie Sie sehen, trifft dies in Ihrem Falle nicht zu. Sollten Sie in einer weiteren Zukunft den Schritt in die Selbständigkeit ins Auge fassen, so darf, analog dem Spitalarzt, erwartet werden, dass Sie sich die nötigen Voraussetzungen sprich Weiterbildungstitel verschaffen werden. Eine detailliertere Beantwortung ist von diesem Punkt an nicht mehr möglich, weil u.a. unklar ist, ob Sie, und wenn ja über welchen Facharzttitel verfügen. 